\title{
INFLUÊNCIA DE VARIÁVEIS AMBIENTAIS NA OCORRÊNCIA DA DENGUE UTILIZANDO GEOPROCESSAMENTO EM TERESINA, PIAUÍ
}

\section{INFLUENCE OF ENVIRONMENTAL VARIABLES IN THE INCIDENCE OF DENGUE USING GEOPROCESSING IN TERESINA, PIAUÍ}

\author{
Gustavo Souza Valladares \\ Doutor em Ciência do Solo pela Universidade Federal Rural do Rio de Janeiro (UFRRJ) \\ Professor Associado da UFPI \\ Pesquisador Produtividade em Pesquisa CNPq - Nível 2 \\ valladares@ufpi.edu.br
}

Izabella Cabral Hassum Doutora em Parasitologia Veterinária pela Universidade Federal Rural do Rio de Janeiro (UFRRJ) Pesquisadora da Embrapa Meio Norte izabella.cabral@hotmail.com

Emanuel Lindemberg Silva Albuquerque Doutor em Geografia pela Universidade Estadual do Ceará (UECE) Professor Adjunto da UFPI lindemberg@ufpi.edu.br

Antônio Carlos dos Santos Mestre em Geografia pela Universidade Federal do Piauí (UFPI) Técnico da SESAPI baulive@hotmail.com

\section{RESUMO}

O objetivo do trabalho foi avaliar a distribuição sazonal da dengue no município de Teresina/PI no ano de 2017, e fazer uma análise correlacionando aspectos ambientais com a distribuição da doença. A área de estudo corresponde a um semicírculo no entorno da área urbana de Teresina, distando $288 \mathrm{~m}$ da região mais periférica, sendo limitada pelo estado do Maranhão a oeste. Foi avaliada a distribuição do número de casos de dengue nos anos de 2015 a 2017. A precipitação pluviométrica e a temperatura do ar foram avaliadas para o ano de 2017. Por meio de sensoriamento remoto e do uso de imagens do Landsat 8 foram estimados os corpos d'água, pelo índice NDWI, e a cobertura vegetal pelo índice NDVI, para os meses de maio a outubro de 2017, contemplando do período chuvoso ao de estiagens. Os resultados demonstraram que a maior incidência da dengue no primeiro semestre dos anos é reflexo de condições ideais da temperatura do ar, da maior precipitação pluviométrica, que reflete na maior superfície ocupada por corpos d'água e maior cobertura vegetal, favorecendo o desenvolvimento do vetor e da doença. Os resultados obtidos com a presente pesquisa indicam claramente a ocorrência da doença correlacionada com fatores ambientais e a sua sazonalidade. A análise dessa pesquisa pode ajudar o poder público no controle do vetor e consequentemente da doença, indicando que no primeiro semestre dos anos deve-se tomar medidas mais efetivas no controle populacional do Aedes aegypti.

Palavras-Chave: Dengue. Sensoriamento Remoto. Sazonalidade. NDWI. NDVI.

\begin{abstract}
The objective of the present study was to evaluate the seasonal distribution of dengue in the city of Teresina in the year 2017, and to make an analysis correlating environmental aspects with the distribution of the disease. The study area corresponds to a semicircle around the urban area of Teresina, distant $288 \mathrm{~m}$ from the most peripheral region, being limited on the west by the state of Maranhão. The distribution of the number of dengue cases in the years 2015 to 2017 was evaluated. Rainfall and air temperature were evaluated for the year 2017 .
\end{abstract}

Recebido em: 02/04/2019

Aceito para publicação em: 06/12/2019 
Remote sensing and the use of Landsat 8 images were used to estimate the water bodies, by the NDWI index, and the vegetable land cover by the NDVI index, from May to October 2017 , considering the rainy season and the dry season. The results showed that the higher incidence of dengue in the first semester of the year is a reflection of the ideal conditions of the air temperature, the higher rainfall, which reflects on the larger surface occupied by water bodies and greater vegetation cover, favoring the development of the vector and disease. The results obtained with the present research clearly indicate the occurrence of the disease correlated with environmental factors and their seasonality. The analysis of this research can help the public power in the control of the vector and consequently of the disease, indicating that in the first half of the year must take more effective measures in the population control of Aedes aegypti.

Keywords: Dengue. Remote Sensing. Seasonality. NDWI. NDVI.

\section{INTRODUÇÃO}

A dengue é uma doença febril viral, do grupo das arboviroses, transmitida pelo díptero Aedes aegypti, que provoca sérios problemas em várias regiões do mundo. Metade da população mundial vive sob o risco da doença, que teve um grande crescimento nas últimas décadas (WHO, 2014). Sua forma hemorrágica pode levar a óbito (GUBLER, 2002). No Brasil as arboviroses tem causado muito problemas de saúde pública na última década (LOPES et al., 2018).

Vários aspectos ambientais podem afetar a distribuição espacial da doença, dentre eles pode-se citar o clima, o relevo e alterações no ecossistema (LIMA, MORAES e PEREIRA, 2011; ASHBY et al., 2017). Dentre os aspectos sociais, destaca-se que, o crescimento populacional, o estilo de vida, a concentração populacional e a precariedade dos serviços de saúde podem ser apontados como fatores que também interferem espacialmente (MENDONÇA, SOUZA e DUTRA, 2009; LIMA, MORAES e PEREIRA, 2011; BARBOSA e SILVA, 2015, DELMELLE et al., 2016). A percepção ambiental é outro fator que pode influenciar na incidência da doença (SILVA et al., 2019). A dengue é conhecida como uma doença urbana, sendo mais comum sua ocorrência em centros urbanos ou nas suas periferias (MESSINA et al., 2015; QI et al., 2015; DELMELLE et al., 2016).

Nos anos de 2015 e 2016 houve um aumento significativo no número de casos de dengue no Brasil (BRASIL, 2018), sendo que no estado do Piauí, incluindo a cidade de Teresina, houve um aumento no ano de 2015, e o número de casos permaneceu elevado até o ano de 2017 , porém não nos patamares de 2015 (PIAUÍ, 2018).

No Piauí a dengue tem sua maior incidência em meados do primeiro semestre dos anos, reduzindo o número de casos entre as semanas epidemiológicas 27 a 29 (MONTEIRO et al., 2009; PIAUÍ, 2018). Portanto a incidência da doença tem um comportamento sazonal. O mapeamento e a distribuição espacial de variáveis relacionadas a doenças auxilia o poder público no planejamento e tomada de decisão em aspectos referentes a gestão pública (DIAS et al., 2005). O Estado deve promover qualidade de vida à população, sendo o saneamento básico fundamental nesse processo, pois tal serviço está diretamente relacionado com a prática da democracia e igualdade social (MARCHI, MENDES, 2017). De acordo com o Trata Brasil (2017), somente $21 \%$ da população de Teresina é atendida por serviço de esgotamento sanitário e somente $15 \%$ do esgoto é tratado, estando entre as piores colocações das capitais brasileiras.

Ao considerar o exposto, o objetivo do presente trabalho foi avaliar a distribuição sazonal da dengue no município de Teresina, estado do Piauí, considerando o recorte temporal de 2015 a 2017, bem como correlacionando à análise dos aspectos ambientais com a distribuição espacial da doença em 2017. Para tais análises, utilizou-se de tecnologias como o sensoriamento remoto e o geoprocessamento.

\section{MATERIAL E MÉTODOS}

O estudo foi desenvolvido para a cidade de Teresina/PI e seu entorno, sendo estabelecida uma área tampão de $288 \mathrm{~m}$ do limite do perímetro urbano da cidade, considerando a possibilidade de 
deslocamento do vetor. A área de estudo foi limitada a oeste pelo limite com o estado do Maranhão (Figura 1). Em 2017 Teresina teve sua população estimada em aproximadamente 850 mil habitantes, com uma área de 1.391,981 km². Tem clima tropical com estação seca de inverno (Aw), segundo a classificação de Köppen, com temperaturas médias variando de 26 a $29{ }^{\circ} \mathrm{C}$, e precipitação pluviométrica de $1325 \mathrm{~mm}$ (ANDRADE, 2016).

Para gerar os mapas temáticos dos corpos d'água e de cobertura vegetal foram utilizadas imagens do Landsat 8 sensor OLI, com correções radiométricas e atmosféricas. As cenas utilizadas correspondem a órbita/ponto 219/064 datadas do período chuvoso ao período de estiagens, fazendo um total de seis datas (23/05/2017, 24/06/2017, 26/07/2017, 27/08/2017, 12/09/2017 e 14/10/2017), não foi possível fazer de todos os meses do ano devido a alta cobertura de nuvens sobre a área de interesse. Os corpos d'água foram mapeados pelo Índice de Água por Diferença Normalizada (NDWI - Normalized Difference Water Index), equação 1, e a cobertura vegetal pelo Índice de Vegetação por Diferença Normalizada (NDVI - Normalized Difference Vegetation Index), equação 2, conforme (MCFEETERS, 1996; CARLSON; RIPLEY, 1997).

$$
\mathrm{NDWI}=\frac{(V E R D-I V P)}{(V E R D+I V P)} \quad \text { Equação } 1 .
$$

Onde: $\mathrm{NDWI}=$ Índice de Água por Diferença Normalizada; VERD = banda na faixa do visível (banda 3) do sensor OLI; IVP = banda na faixa do infravermelho próximo do sensor OLI (banda 5).

$$
\mathrm{NDVI}=\frac{(I V P-V E R M)}{(I V P+V E R M)} \quad \text { Equação } 2 .
$$

Onde: NDVI = Índice de Vegetação por Diferença Normalizada; IVP = banda na faixa do infravermelho próximo do sensor OLI (banda 5); VERM = banda na faixa do visível (banda 4) do sensor OLI.

Figura 1 - Área de estudo considerada para avaliação da dinâmica dos corpos d'água e da cobertura vegetal em Teresina/PI

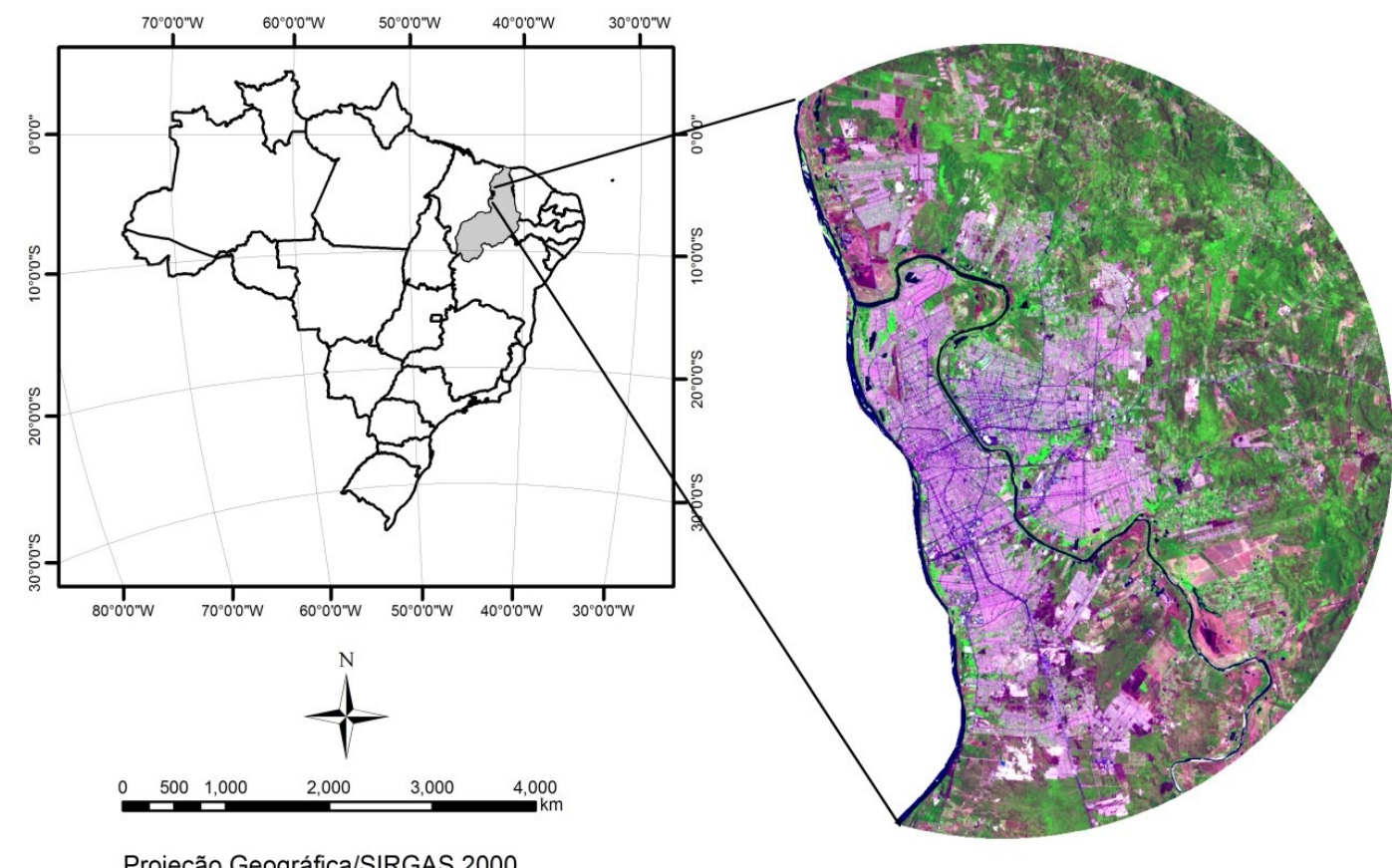

Projeção Geográfica/SIRGAS 2000 
Os dados de precipitação e temperatura foram obtidos junto ao Instituto Nacional de Meteorologia (INMET), considerando os valores mensais para o ano de 2017. A distribuição do número de casos de dengue para o município de Teresina, nos anos de 2015 a 2017, foi obtido junto ao Sistema de Informação de Agravos de Notificação (SINAN), do Ministério da Saúde.

Os números de casos de dengue das semanas epidemiológicas de 2017, correspondentes as datas dos dados iconográficos, foram correlacionados e efetuadas análises de regressão com os resultados obtidos com o NDWI, o NDVI, com a precipitação mensal, e as temperaturas médias, mínimas e máximas mensais. Metodologia semelhante de correlação espacial dos casos de dengue com fatores socioambientais, socioeconômicos e demográficos utilizando análise de regressão encontrada nos trabalhos Almeida, Medronho e Valença (2017) e Almeida e Da Silva (2018).

\section{RESULTADOS E DISCUSSÃO}

Os casos de dengue no município de Teresina, estado do Piauí, entre os anos de 2015 a 2017 podem ser observados na figura 2. Observa-se maior frequência de casos no primeiro semestre do ano comportamento também identificado na pesquisa de Rodrigues, Costa e Lima (2018) em Uberlândia, geralmente havendo aumento a partir da sétima semana epidemiológica e redução a partir da vigésima sétima semana epidemiológica. Em 2015 foram registrados 5045 casos, contra 3157 em 2016 e 3109 em 2017.

Figura 2 - Distribuição do número de casos de dengue por semana epidemiológica no município de Teresina/PI nos anos de 2015 a 2017

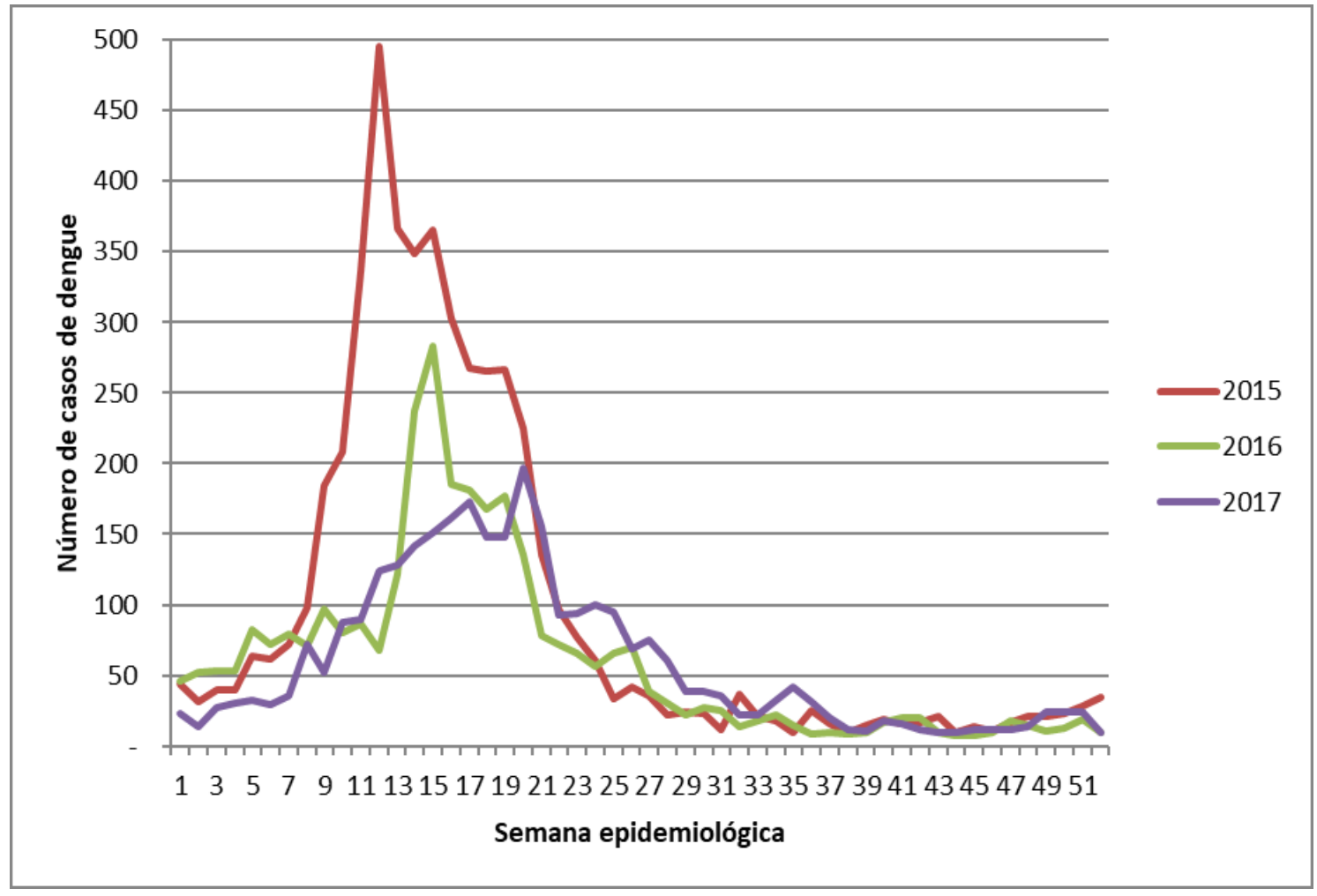

A distribuição espacial dos casos de dengue nos bairros da cidade de Teresina para 2017 é apresentada na Figura 3. Observa-se grande variabilidade da ocorrência da doença na cidade, que apresenta vários bairros sem nenhuma incidência da doença e bairros que passam de 100 casos. As porções mais a oeste e o norte concentram os maiores números de casos, coincidindo com os principais rios que drenam a cidade, no caso Rios Parnaíba e Poti. 
Figura 3 - Distribuição do número de casos de dengue por bairro no município de Teresina/PI no ano de 2017

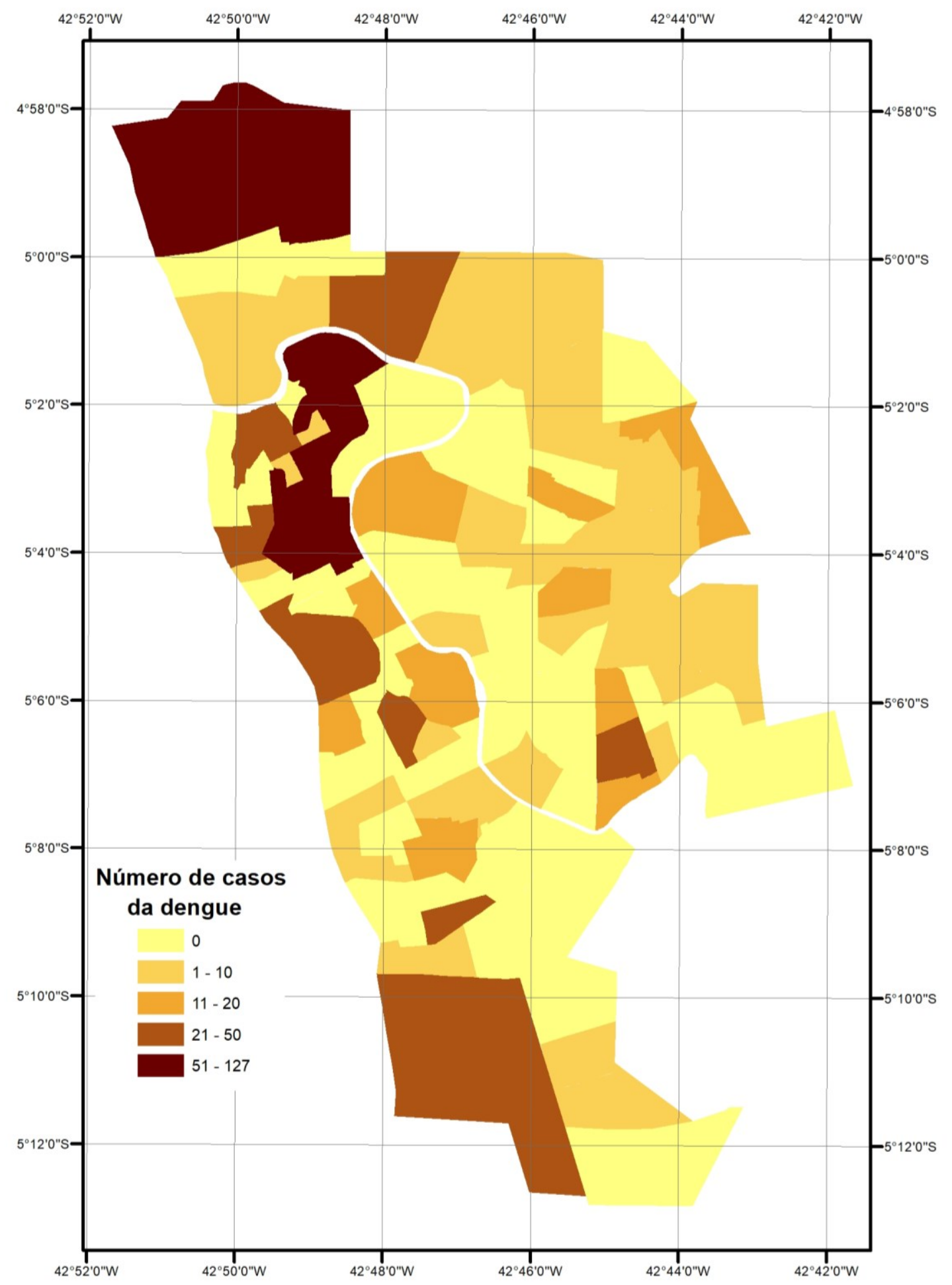

As imagens do Landsat 8 , sensor OLI, de maio e setembro de 2017 podem ser observadas na figura 4, foram selecionadas como exemplos de período chuvoso e de estiagens. A área de estudo corresponde a $585,1 \mathrm{~km}^{2}$ no entorno do perímetro urbano de Teresina. Maio corresponde ao período 
chuvoso e de temperaturas menores (Figura 5) e setembro representa o período de estiagem e com temperaturas mais elevadas.

Figura 4 - Recorte da cena referente a órbita/ponto 219/064 de maio e setembro de 2017, referente ao perímetro urbano e entorno de Teresina/PI. Composição falsa cor RGB das bandas 654
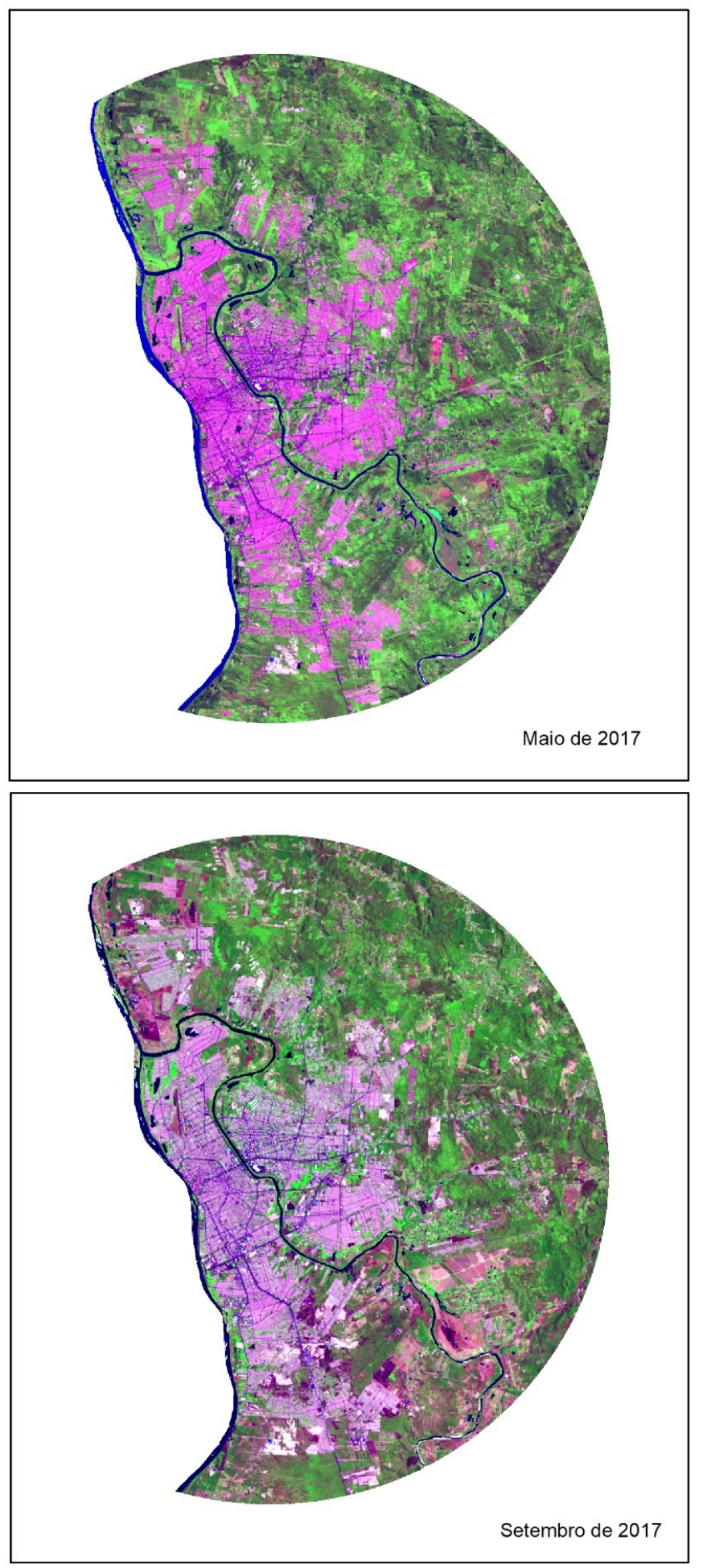
Figura 5 - Distribuição mensal da precipitação e da temperatura média em Teresina/PI no ano de 2017.

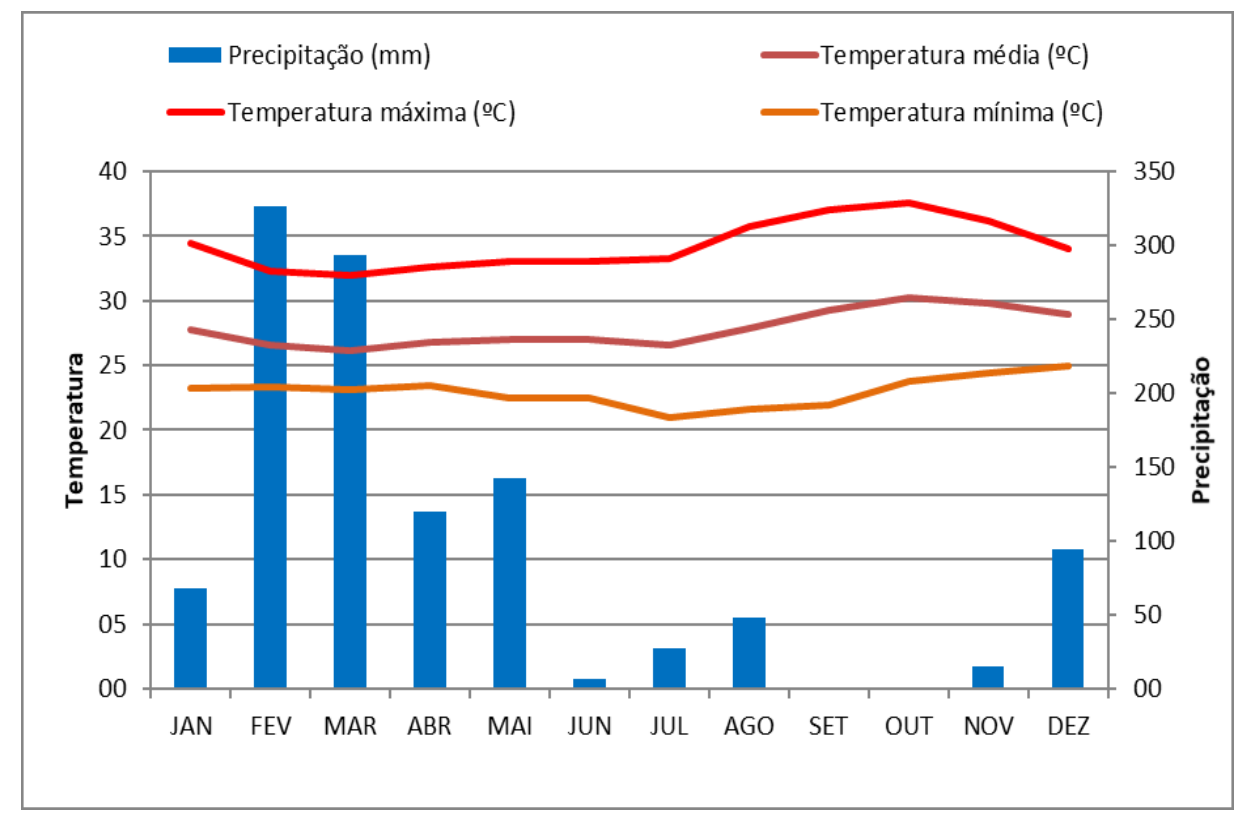

Fonte: INMET.

Os resultados demonstram uma redução significativa na lâmina d'água na área de estudo, sendo que de maio para setembro a redução foi de $19 \%$, utilizando o mês de maio como referência (Figura 6 e Tabela 1). A cobertura vegetal teve comportamento semelhante com redução de maio para setembro de 18\% (Figura 7 e Tabela 1). Deve-se destacar que parte dos corpos d'água podem ter sido mapeados como vegetação, pois pode haver uma cobertura por plantas aquáticas na superfície das águas, e seu comportamento é semelhante ao da vegetação verde, quando se utiliza o NDVI.

A maior lâmina d'água em maio comparado a setembro (Figura 6 e Tabela 1) reflete a maior precipitação pluviométrica ocorrida entre os meses de fevereiro a maio (Figura 5), sendo que a maior cobertura vegetal também é observada em maio (Figura 7 e Tabela 1). Neste período choveu 882 $\mathrm{mm}$, o que corresponde a $77 \%$ do volume de chuva no ano de 2017 . A maior quantidade de corpos d'água favorece o acúmulo de água e, com isso, a proliferação do vetor, no caso o $\mathrm{A}$. aegypti.

Tabela 1 - Distribuição da lâmina d'água e da cobertura vegetal em $\mathrm{km}^{2}$ em Teresina/PI, para alguns meses do ano de 2017

\begin{tabular}{lcccccc}
\hline Classe de cobertura & Maio & Junho & Julho & Agosto & Setembro & Outubro \\
\hline Lâmina d'água & 11,6 & 9,4 & 8,7 & 8,3 & 9,4 & 7,6 \\
Cobertura vegetal & 475,8 & 464,7 & 440,4 & 418,0 & 391,7 & 351,9 \\
\hline
\end{tabular}

Segundo Beserra et al. (2006), trabalhando com diferentes regiões bioclimáticas do estado da Paraíba, a temperatura favorável ao desenvolvimento de $A$. aegypti encontra-se entre $21^{\circ} \mathrm{C}$ e $29^{\circ} \mathrm{C}$, e para a longevidade e fecundidade dos adultos, entre $22^{\circ} \mathrm{C}$ e $30^{\circ} \mathrm{C}$. Portanto, conforme a figura 4 , entre os meses de fevereiro e julho há condições de temperatura do ar muito favoráveis para o vetor da dengue. Nessas condições podem ocorrer mais de 20 gerações do vetor (BESERRA et al., 2006), favorecendo a proliferação da doença.

Os resultados encontrados para Teresina/PI são corroborados com as análises realizadas em Cáceres-MT (LIMA; MORAES; PEREIRA, 2011), em que períodos com aumento dos corpos d'água e nessa faixa de temperatura favorecem a ocorrência da doença. De acordo com Mendonça (2003), a temperatura interfere na distribuição do vetor, na quantidade de picadas e no período de incubação. 
Ao levar em consideração os ciclos biológicos do vetor, e conforme os trabalhos de Beserra et al. (2006) e Lima; Moraes; Pereira (2011), nas condições de temperatura de Teresina, um mosquito pode causar a infecção em até 45 dias após o seu nascimento. Portanto as ótimas condições de temperatura, o aumento dos corpos d'água e da cobertura vegetal verde no primeiro semestre do ano, são importantes fatores na explosão do número de casos de dengue nesse período do ano, justificando a sazonalidade da doença.

Figura 6 - Distribuição espacial da lâmina d'água em Teresina/PI nos meses de maio e setembro de 2017. Fonte: Landsat 8 / sensor OLI
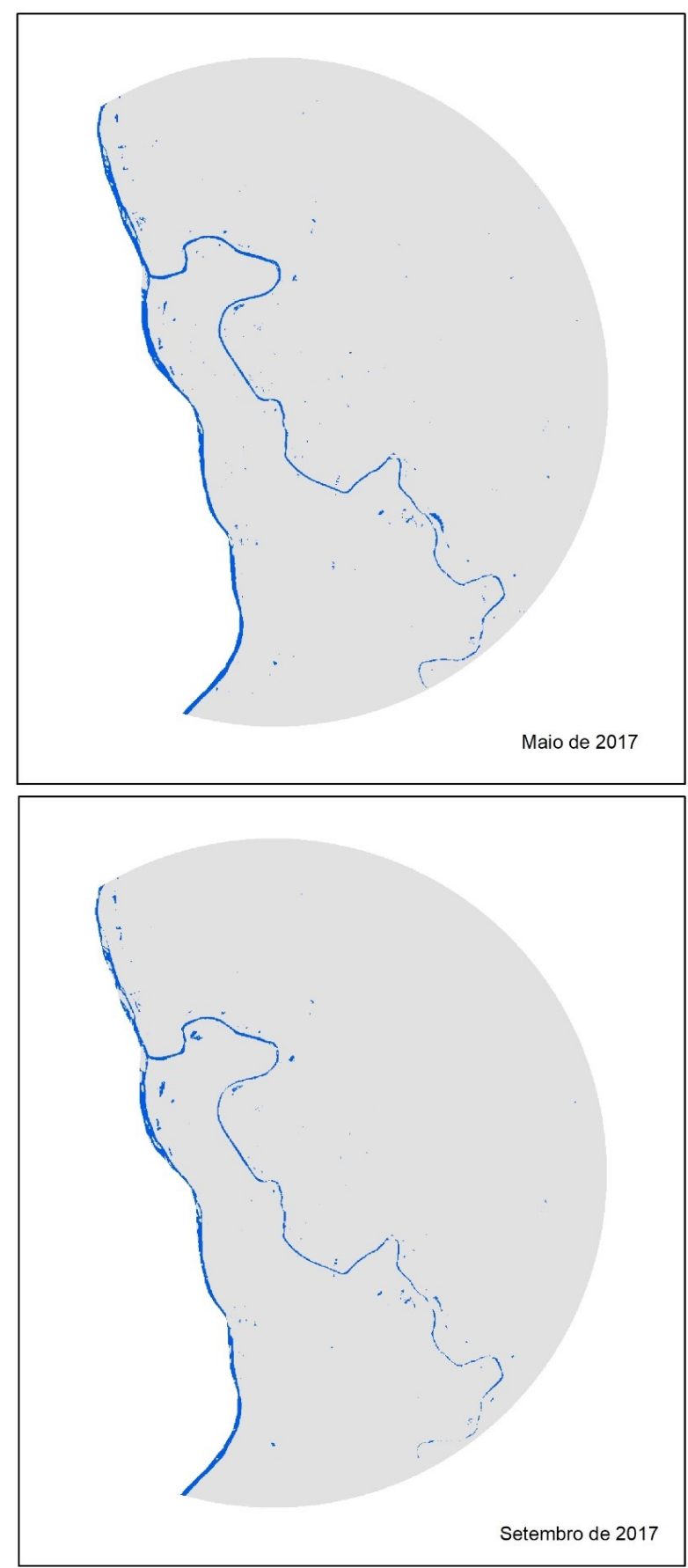
Figura 7 - Distribuição espacial da cobertura vegetal em Teresina/PI nos meses de maio e setembro de 2017. Fonte: Landsat 8/sensor OLI
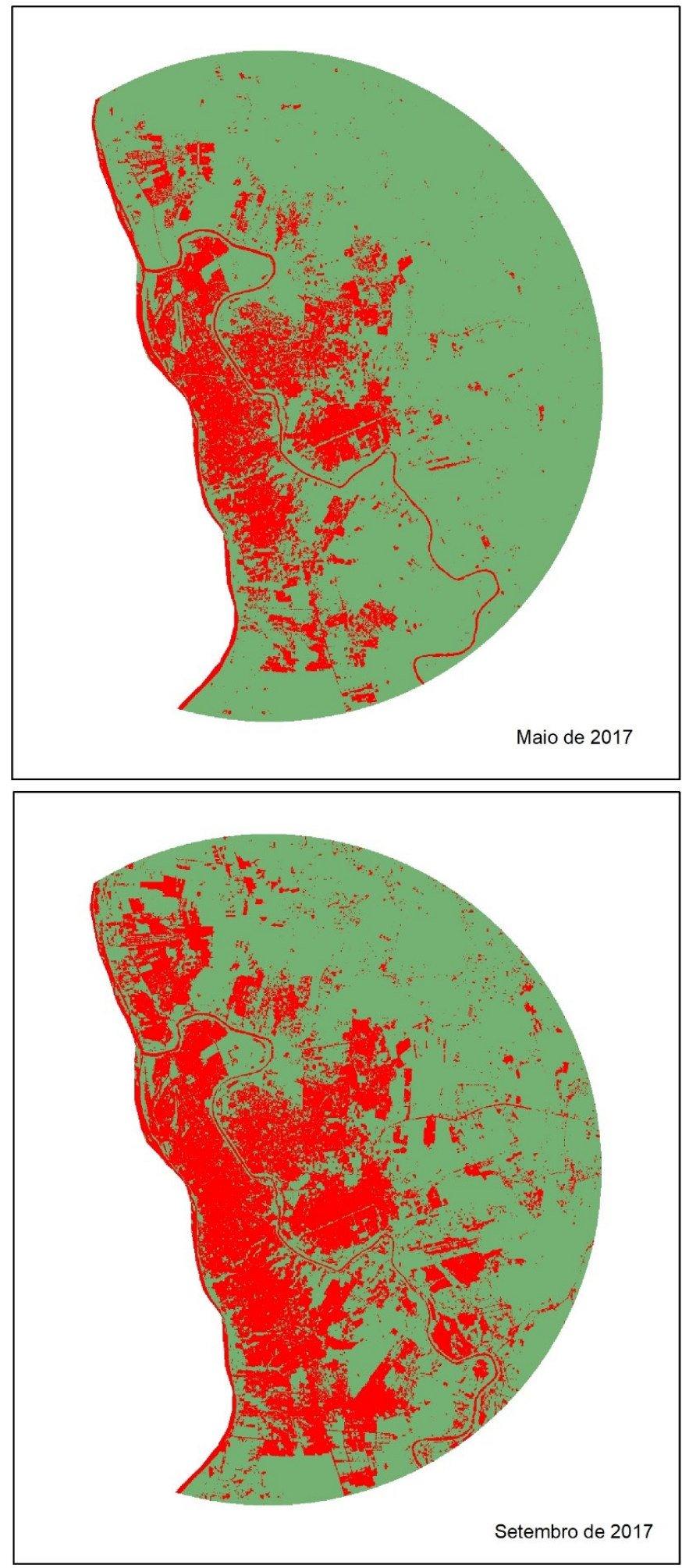
Foram efetuadas análises de correlação linear simples do número de casos de dengue nas semanas epidemiológicas e os resultados de sensoriamento remoto e dados climáticos (Tabela 2.). Os resultados indicam correlação significativa, positiva e elevada entre lâmina d'água, cobertura vegetal e precipitação pluviométrica, sendo que apesar das duas primeiras variáveis serem reflexo da precipitação pluviométrica, as correlações com os indicadores obtidos por NDWI e NDVI foram muito eficientes, pois tiveram valores de coeficiente de correlação mais elevados. Ashby et al. (2017), utilizando sensoriamento remoto e geoprocessamento também obteve resultados positivos no estudo da dengue. Portanto se mostrando excelentes indicadores da ocorrência da doença. Já temperatura média e máxima tiveram correlação negativa e significativa, menos elevada, indicando que maiores temperaturas reduzem o número de casos da dengue, pois os valores vão se aproximando do limite do conforto térmico para os vetores. As temperaturas mínimas não tiveram correlação com o número de casos da dengue.

Tabela 2 - Correlação linear simples ( $r$ ) entre o número de casos de dengue na semana epidemiológica com as variáveis ambientais lâmina d'água, cobertura vegetal, temperatura média do ar, temperatura máxima do ar, temperatura mínima do ar e precipitação pluviométrica em Teresina/PI, para seis meses do ano de 2017

\begin{tabular}{ccccccc}
\hline Variáveis & água & vegetação & TMEDIA & TMAX & TMIN & PPT \\
\hline dengue & $0,87^{*}$ & $0,84^{*}$ & $-0,58^{*}$ & $-0,76^{*}$ & $0,39 \mathrm{~ns}$ & $0,82^{*}$ \\
\hline
\end{tabular}

Água=lâmina d'água, vegetação=cobertura vegetal, TMEDIA=temperatura média do ar, TMAX=temperatura máxima do ar, TMIN=temperatura mínima do ar e PPT=precipitação pluviométrica. * - significativo a $5 \%$ de probabilidade, ns - não significativo.

As análises de regressão detalharam o comportamento entre os casos de dengue e as variáveis ambientais. (Figura 8).

A figura 8a indica comportamento linear diretamente proporcional do número de casos de dengue com a lâmina d'água, com coeficiente de determinação de $R^{2}$ de 0,76 , indicando que $76 \%$ dos dados correspondem ao modelo linear proposto, ou seja, o aumento de $1 \mathrm{~km}^{2}$ de lâmina d'água corresponde ao aumento de 34 casos da doença aproximadamente. A regressão entre o número de casos de dengue e a cobertura vegetal (Figura $8 b$ ) teve um ajuste ao modelo polinomial quadrático muito bom, com $\mathrm{R}^{2}$ de 0,92 , indicando um efeito positivo entre o aumento da cobertura vegetal e o número de casos da doença. Qi et al. (2015) estudando fatores ambientais que interferem na incidência da dengue na China também observaram relação não linear entre o NDVI e a doença, corroborando com o presente trabalho. As temperaturas médias e máximas mensais (Figura $8 \mathrm{c} \mathrm{e} \mathrm{d}$ ) indicam efeito inverso ao número de casos da doença para a cidade de Teresina, isto é, os meses de menores temperaturas favorecem a maior ocorrência da doença, porém os coeficientes de determinação não foram tão elevados, indicando ajustes dos modelos matemáticos inferiores aos das variáveis obtidas por sensoriamento remoto. A precipitação pluviométrica (Figura 8e) voltou a ter efeito positivo com os casos da doença, com ajuste do modelo linear, mais com $\mathrm{R}^{2}$ inferior aos apresentados para lâmina d'água e cobertura vegetal. 
Figura 8 - Diagramas de dispersão e análise de regressão entre os casos de dengue e as variáveis ambientais
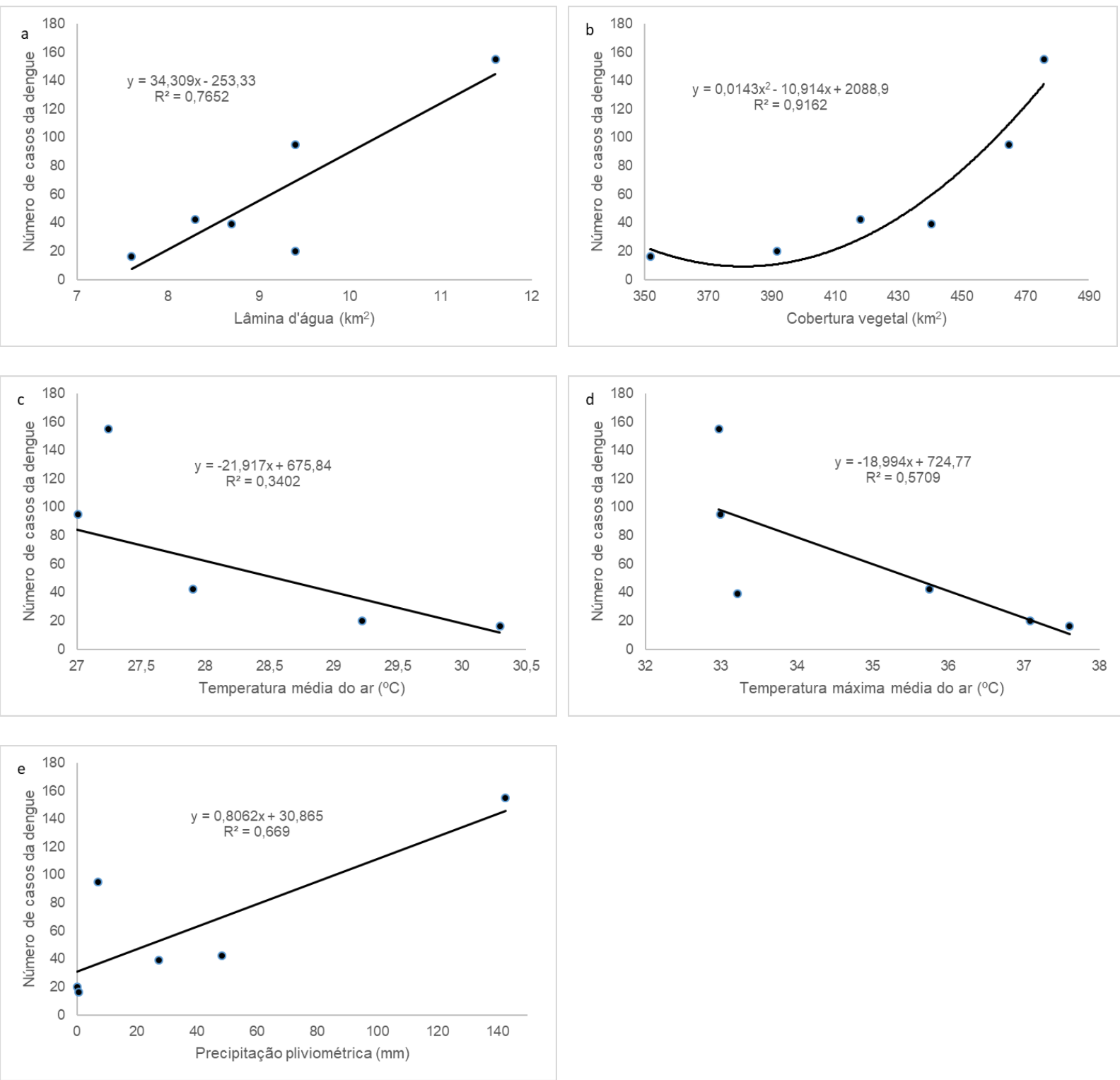

\section{CONCLUSÕES}

As geotecnologias, no caso o sensoriamento remoto com uso de imagens óticas orbitais e sua aplicação por geoprocessamento, gerando os índices NDWI e NDVI, foram eficientes, respectivamente, no mapeamento dos corpos d'água e cobertura vegetal para o recorte espacial adotado no estudo em epígrafe, indicando uma maior ocorrência dessas classes no primeiro semestre de 2017. E se mostraram indicadores promissores para a ocorrência da doença, com correlação e ajuste a modelos matemáticos superiores aos dados de chuva e temperatura do ar.

Destaca-se que essas informações, aliadas a análise da temperatura do ar, explica o maior número de casos de dengue no primeiro semestre do ano. Portanto, para reduzir os casos de dengue na cidade de Teresina/PI, o poder público municipal deve, no primeiro semestre de cada ano, centrar 
esforços nas medidas de maior eficácia no controle da população de Aedes aegypti e albopictus, uma vez que é possível identificar, espacialmente, os setores mais favoráveis à proliferação do vetor.

\section{AGRADECIMENTOS}

Os autores agradecem ao Programa Pesquisa para o SUS: gestão compartilhada em saúde (PPSUS), no âmbito do Edital FAPEPI/MS-DECIT/CNPq/SESAPI N ${ }^{\circ} 002 / 2016$, pelo financiamento do projeto intitulado Avaliação geoespacial da ocorrência de arboviroses no estado do Piauí: um enfoque na dengue e no zika vírus, bem como a Gerência de Vigilância em Saúde da Secretaria de Estado da Saúde do Piauí (SESAPI), pela parceria de trabalho. Ao CNPq pela Bolsa de Produtividade em Pesquisa do primeiro autor Proc. 301254/2017-6.

\section{REFERÊNCIAS}

ALMEIDA, A. S., MEDRONHO, R. A., VALENCIA, L. I. O. Análise espacial da dengue e o contexto socioeconômico no município do Rio de Janeiro, RJ. Revista de Saúde Pública, v. 43, p. 666-673, 2009. https://doi.org/10.1590/S0034-89102009000400013

ALMEIDA, C. P.; DA SILVA, R. M. Análise da ocorrência dos casos de dengue e sua relação com as condições socioambientais em espaços urbanos: os casos de João Pessoa, Cabedelo e Bayeux, no estado da Paraíba-Brasil. Hygeia, v. 14, n. 26, p. 56-79, 2018.

ANDRADE, C.S. Teresina e clima: indissociabilidades no estudo da cidade. Revista Equador, v. 5, n. 3, p. 398-420, 2016.

ASHBY, J.; MORENO-MADRIÑÁN, M.; YIANNOUTSOS, C.; STANFORTH, A. Niche modeling of dengue fever using remotely sensed environmental factors and boosted regression trees. Remote Sensing, v. 9, n. 4, p. 1-15, 2017. https://doi.org/10.3390/rs9040328

BARBOSA, I.R.; SILVA, L.P. Influência dos determinantes sociais e ambientais na distribuição espacial da dengue no município de Natal-RN. Revista Ciência Plural, V. 1, N. 3, P. 62-75, 2015.

BESERRA, E.B.; CASTRO JR, F.P.D.; SANTOS, J.W.D.; SANTOS, T.D.S.; FERNANDES, C.R. Biology and thermal exigency of Aedes aegypti (L.)(Diptera: Culicidae) from four bioclimatic localities of Paraiba. Neotropical Entomology, v. 35, n. 6, p. 853-860, 2006. https://doi.org/10.1590/S1519$\underline{566 \times 2006000600021}$

BRASIL. MINISTÉRIO DA SAÚDE - MS. Secretaria de Vigilância em Saúde. Monitoramento dos casos de dengue, febre de chikungunya e febre pelo vírus Zika até a Semana Epidemiológica 10 de 2018. Boletim Epidemiológico, v.49, n.14, p.1-13. 2018.

CARLSON, T.N.; RIPLEY, D.A. On the relation between NDVI, fractional vegetation cover, and leaf area index. Remote sensing of Environment, v. 62, n. 3, p. 241-252, 1997. https://doi.org/10.1016/S0034-4257(97)00104-1

DELMELLE, E.; HAGENLOCHER, M.; KIENBERGER, S.; CASAS, I. A spatial model of socioeconomic and environmental determinants of dengue fever in Cali, Colombia. Acta tropica, v., 164, p. 169-176, 2016. https://doi.org/10.1016/i.actatropica.2016.08.028

DIAS, N.W.; NASCIMENTO, L.F.C.; BATISTA, G.T.; SOUZA CATELANI, C. Inferências espaciais sobre Saúde Pública e Desenvolvimento no Vale do Paraíba Paulista. Revista Brasileira de Gestão e Desenvolvimento Regional, v. 2, n. 2, 2005.

GUBLER, D.J. Epidemic dengue/dengue hemorrhagic fever as a public health, social and economic problem in the 21st century. Trends in microbiology, v. 10, n. 2, p. 100-103, 2002. https://doi.org/10.1016/S0966-842X(01)02288-0

LIMA, S.F.S.; MORAES, E.C.; PEREIRA, G. Analysis of the influence of environmental variables obtained from satellite data on the increase of dengue in the municipality of Cáceres (Mato Grosso State, Brazil) in 2009. GEOGRAFIA, Rio Claro, v. 36, Número Especial, p. 135-142. 2011. 
LOPES, T. R. R.; SILVA, C. S.; PASTOR, A. F.; SILVA JÚNIOR, J. V. J. Dengue in Brazil in 2017: what happened?. Revista do Instituto de Medicina Tropical de São Paulo, v. 60, 2018. https://doi.org/10.1590/s1678-9946201860043

MARCHI, C.M.D.F.; MENDES, V.L.P.S. Gestão do saneamento básico: democracia representativa e participação social em um município da região metropolitana de Salvador-Bahia. Revista Brasileira de Gestão e Desenvolvimento Regional, v. 14, n. 1, p.110-134. 2018.

MCFEETERS, S.K. The use of the Normalized Difference Water Index (NDWI) in the delineation of open water features. International journal of remote sensing, v. 17, n. 7, p. 1425-1432, 1996. https://doi.org/10.1080/01431169608948714

MENDONÇA, F. A. Aquecimento global e saúde: uma perspectiva geográfica - notas introdutórias. Revista Terra Livre, n. 20, p. 205-221, 2003.

MENDONÇA, F.A.; VEIGA E SOUZA, A.; ALMEIDA DUTRA, D. Saúde pública, urbanização e dengue no Brasil. Sociedade \& natureza, v. 21, n. 3, 2009. https://doi.org/10.1590/S1982$\underline{45132009000300003}$

MESSINA, J. P.; BRADY, O. J.; PIGOTT, D. M.; GOLDING, N.; KRAEMER, M. U.; SCOTT, T. W.; WINT, G.W.; SMITH, D.L.; HAY, S. I. The many projected futures of dengue. Nature Reviews Microbiology, v. 13, n. 4, p. 230. 2015. https://doi.org/10.1038/nrmicro3430

MONTEIRO, E.S.C.; COELHO, M.E.; CUNHA, I.S.D.; CAVALCANTE, M.D.A.S.; CARVALHO, F.A.D.A. Aspectos epidemiológicos e vetoriais da dengue na cidade de Teresina, Piauí-Brasil, 2002 a 2006. Epidemiol serv saúde, v.18, n.4, p.365-374. 2009. https://doi.org/10.5123/S1679$\underline{49742009000400006}$

PIAUÍ. SECRETARIA DE ESTADO DA SAÚDE DO PIAUÍ - SESAPI. Dengue, Chikungunya, Zika e Microcefalia. Boletim da $14^{a}$ Semana Epidemiológica. Teresina: Secretaria de Estado da Saúde do Piauí. 5 p. 2018.

QI, X.; WANG, Y.; LI, Y.; MENG, Y.; CHEN, Q.; MA, J.; GAO, G. F. The effects of socioeconomic and environmental factors on the incidence of dengue fever in the Pearl River Delta, China, 2013. PLoS neglected tropical diseases, v. 9, n. 10, e0004159. 2015. https://doi.org/10.1371/journal.pntd.0004159

RODRIGUES, E. A. S.; COSTA, I. M.; LIMA, S. C. Epidemiologia da dengue, zika e chikungunyia, entre 2014 a 2016, em Uberlândia (MG). Hygeia - Revista Brasileira de Geografia Médica e da Saúde 14 (30): 62 - 81, Dezembro/2018. Disponível em http://www.seer.ufu.br/index.php/hygeia

SILVA, A.V.B.A.; BRANDÃO, C.R.P.; VITÓRIA, N.S. Percepção ambiental acerca da tríplice epidemia (dengue-chikungunya-zika) e sua relação com os resíduos sólidos. Semioses, v. 13, n. 2, p. 13-27. 2019. https://doi.org/10.15202/1981996x.2019v13n2p13

TRATA BRASIL. Ranking do Saneamento Instituto Trata Brasil 2017. São Paulo. GO Associados/Instituto Trata Brasil. 122p. 2017. Disponível em http://tratabrasil.org.br/datafiles/estudos/ranking/2017/relatorio-completo.pdf.

WORLD HEALTH ORGANIZATION. Dengue and severe dengue. 2014. 4p. 